revised to ApJ

\title{
Thermal Radiation from GRB Jets
}

\author{
Akira Mizuta ${ }^{1,2}$, Shigehiro Nagataki ${ }^{3}$, and Junichi $\mathrm{Aoi}^{3}$ \\ AM, e-mail: mizuta@post.kek.jp
}

\begin{abstract}
In this study, the light curves and spectrum of the photospheric thermal radiation from ultrarelativistic gamma-ray burst (GRB) jets are calculated using 2D relativistic hydrodynamic simulations of jets from a collapsar. As the jet advances, the density around the head of the jet decreases, and its Lorentz factor reaches as high as 200 at the photosphere and 400 inside the photosphere. For an on-axis observer, the photosphere appears concave shaped due to the low density and high beaming factor of the jet. The luminosity varies because of the abrupt change in the position of the photosphere due to the internal structure of the jet. Comparing our results with GRB090902B, the flux level of the thermal-like component is similar to our model, although the peak energy looks a little bit higher (but still within a factor of 2). From the comparison, we estimate that the bulk Lorentz factor of GRB090902B is $\Gamma \sim 2.4 \times 10^{2}\left(r / 10^{12} \mathrm{~cm}\right)$ where $r$ is the radius of the photosphere. The spectrum for an on-axis observer is harder than that for an off-axis observer. There is a time lag of a few seconds for high energy bands in the light curve. This may be the reason for the delayed onset of $\mathrm{GeV}$ emission seen in GRB080916C. The spectrum below the peak energy is a power law and the index is $2.3 \sim 2.6$ which is softer than that of single temperature plank distribution but still harder than that of typical value of observed one.
\end{abstract}

Subject headings: gamma-ray burst: individual (GRB090902B, GRB080916C) - hydrodynamics — methods: numerical — radiation mechanisms: thermal

\footnotetext{
${ }^{1}$ Theory Center, Institute of Particle and Nuclear Studies, KEK (High Energy Accelerator Research Organization), 1-1 Oho, Tsukuba 305-0801, Japan

${ }^{2}$ Center for Frontier Science, Chiba University Yayoi-cho 1-33, Inage-ku, Chiba, 263-8522, Japan

${ }^{3}$ Yukawa Institute for Theoretical Physics, Kyoto University, Kitashirakawa Oiwake-cho, Sakyo-ku, Kyoto, 606-8502, Japan
} 


\section{INTRODUCTION}

Gamma-ray bursts (GRBs), the most energetic explosion in the universe, show a nonthermal spectrum, implying that GRBs originate from an optically thin region (e.g. Piran (1999) and references therein). On the other hand, some long GRBs are associated with a supernova (SN) explosion (e.g., Woosley and Bloom (2006) and references therein). Are GRBs already optically thin when they break out the their progenitors ? The answer is obviously no, since the jet density should be high and Lorentz factor should be low. Thus, we can expect to observe the thermal radiation from the photosphere prior to GRBs. Even in the prompt phase, there may be a thermal radiation component coming from the photosphere.

About 25 years ago, it was suggested GRBs should show thermal spectra unless they are coming from relativistically-moving objects (Goodman 1986). Similar to our motivation, the thermal radiation from the photosphere of GRBs were analytically discussed in some papers (Blinnikov et al. (1999), Ryde (2005), Pe'er et al. (2007), Li (2007), and Pe'er (2008)). In the photospheric model Lazzati et al. (2009), thermal radiation is scattered by relativistic electrons producing non-thermal photons of GRBs (e.g. Rees and Mészáros (1999); Ioka et al. (2007); Toma et al. (2009 and 2010)). The effects of magnetic fields, for example the reconnection, on the emission have been discussed in some papers (Giannios (2006) and Zhang \& Pe'er (2009)).

Thermal components have been observed in some GRBs from the data obtained from BATSE catalog and/or BeppoSAX catalog (Ghirlanda et al. (2007), and Ryde \& Pe'er (2009)). A thermal component has been reported for GRB060218 associated with SN2006aj (Campana et al 2006; Liang et al. 2006). Recently, Fermi Gamma-ray Space Telescope observed GRB090902B, showing a clear spectrum consist of two components (Ryde et al. 2010) with one appearing to be a thermal component.

In this study, we investigate the thermal radiation from the photosphere of GRBs through numerical simulations. Some studies have been carried out on the jet propagation of GRBs (e.g. Mizuta \& Aloy (2009) and references therein); however these studies did not determine the photosphere's location and the number of thermal photons radiated from GRBs except for Lazzati et al. (2009). In this study, we determine the location and shape of the photosphere of GRBs as a function of time and estimate the light curve and spectrum of the thermal radiation. Here we present thermal spectrum and light curves for different viewing angles and light curves for several energy bins corresponding to Fermi and Swift data. We also discuss the unique spectrum of GRB090902B and show the delayed onset of hard photons possibly related to the one seen in GRB080916C (Abdo et al. 2009), and we discuss viewing angle effects on short GRBs and X-ray flash (X-ray flare). 


\section{NUMERICAL SETUP}

This section describes the progenitor model and the numerical conditions of the hydrodynamic calculations. Although the structure of the progenitor at the pre-SN stage is not known, realistic calculations of stellar evolution of massive stars have been recently done by Woosley \& Heger (2006), Yoon \& Langer (2005) and Yoon et al. (2006). We choose one of models in Woosley \& Heger (2006). The model is named 16TI whose core has a relatively high spin at the pre-SN stage; this progenitor model is also used by Morsony et al. (2007), Lazzati et al. (2009) and Morsony et al. (2010). At zero age, the progenitor is assumed to have a mass of 16 solar masses and low metallicity $\left(0.01 Z_{\odot}\right)$. At the pre-SN stage, the total mass is 13.95 solar masses and the progenitor radius $\left(r_{*}\right)$ is $4 \times 10^{10} \mathrm{~cm}$.

The 2D spherical coordinate system $(r \times \theta)$ is used for hydrodynamic calculation, assuming axisymmetry and equatorial plane symmetry. The computational domain covers the region of $10^{9} \mathrm{~cm} \leq r \leq 3 \times 10^{12} \mathrm{~cm}$ and $0^{\circ} \leq \theta \leq 90^{\circ}$. We remap the density profile from the progenitor model into the computational domain $\left(r_{\min } \leq r \leq r_{*}\right)$, assuming spherical symmetry. The reflective boundary condition is applied at the polar axis and the equatorial plane. Since the stellar wind theory is not well known so far and a weak wind is expected due to very low metallicity, we assume the gas outside the progenitor is dilute and the power law distribution $\rho=1.8 \times 10^{-14}\left(r / r_{*}\right)^{2} \mathrm{~g} \mathrm{~cm}^{-3}$. Since this weak wind does not affect to the jet dynamics and the opacity, the model corresponds to the most luminous case.

The radial grid consists of $N_{r}=2640$ points, uniformly spaced in $\log r$, extending from $r_{\min }=10^{9} \mathrm{~cm}$ to $r_{\max }=3 \times 10^{12} \mathrm{~cm}$. The smallest radial grid spacing, at $r_{\min }$, is $\Delta r_{\min }=10^{7} \mathrm{~cm}$, while the largest one, at $r_{\max }$, is $\Delta r_{\max }=7.6 \times 10^{9} \mathrm{~cm}$. The uniform 120 polar grid is spaced between $0^{\circ} \leq \theta \leq 30^{\circ}$, i.e., $\Delta \theta=0.25^{\circ}$. The 60 uniform logarithmic grids are spaced in the range of $30^{\circ} \leq \theta \leq 90^{\circ}$.

The mechanism of the jet formation near the central black hole is still under debate (but see e.g., Nagataki et al. (2007), Nagataki (2009)). A very hot, relativistic and temporary constant jet is injected from the inner most grid, i.e., $r=r_{\min }$ with a half opening angle $10^{\circ}$. The velocity vector is parallel to the radial unit vector. The luminosity of the injected jet is $5.5 \times 10^{50} \mathrm{erg} / \mathrm{s}$. Lorentz factor $\left(\Gamma_{0}\right)$ is 5 and specific internal energy $\left(\epsilon_{0} / c^{2}\right)$ is 80 , where $c$ is the speed of light. This jet has a potential to be accelerated to a Lorentz factor of more than 500, applying relativistic Bernoulli's principle ; $h \Gamma=$ const, along a stream line $\left(h_{0} \Gamma_{0}=538\right.$ in our case), where $h$ is specific enthalpy, if all thermal energy is converted to kinetic energy without dissipation. We follow the jet propagation until the head of the jet reaches $r=3 \times 10^{12} \mathrm{~cm}$.

A special relativistic hydrodynamic code developed by one of the authors (AM Mizuta et al. 
(2004) and Mizuta et al. (2006)) is used for hydrodynamic simulations. The version with 2nd order accuracy both in time and space is used, including some dissipation to prevent numerical oscillation at strong shocks.

\section{RESULTS}

\subsection{Hydrodynamics}

In the early phase of the evolution, the jet drills through the progenitor envelope, keeping good collimation, as shown in previous numerical simulations by Alov et al. (2000), Zhang et al. (2003, 2004), Mizuta et al. (2006), Morsony et al. (2007), Mizuta \& Aloy (2009), and Lazzati et al. (2009). The forward shock drives the envelopes to the high pressure and high temperature. A cocoon originates from the jet material because of the reverse shock at the head of the jet and surrounds the jet. Since the density of the jet is considerably lower than that of the stellar envelopes, a fast backflow and some vortices appear in the cocoon (Mizuta et al. 2010). Internal shocks in the jet appear due to interaction between the jet and the high pressure cocoon and backflows (Komissarov \& Falle $(1997,1998)$ and Morsony et al. $(2007))$. The jet reaches the progenitor surface at about $t_{\text {lab }}=7 \mathrm{~s}$.

When the shock breaks out the progenitor surface, the components near the head of the jet starts expanding, resulting in a high Lorentz factor component $(\Gamma \sim 200)$ (two top panels of Fig. 1; density and Lorentz factor contours at $t_{\mathrm{lab}}=30 \mathrm{~s}$ ). The shocked envelopes are also expanding into circumstellar matter. Since most of the components of the jet are still surrounded by the expanding cocoon and high density progenitor envelopes, the jet remains collimated. The pressure in the cocoon and envelope decreases as they expand. The components injected from computational boundary $\left(r=r_{\min }\right)$ at a later phase can easily propagate in a radial direction without dissipation (four bottom panels of Fig. 1). The blue regions in density contours and the red and purple regions in Lorentz factor contours are free expanding regions. The Lorentz factor exceeds 400. This free expansion ends at the internal shock. On the other hand, since the velocity of the expanding cocoon and stellar envelopes is less than its sound speed $(\sim 0.5 c)$, it is delayed with the head of the jet. As a result, the head of the jet is quite relaxed, resulting in a high Lorentz factor component $(\Gamma \sim 200)$. The internal structures imprinted in the jet before the shock break still remain near the head of the jet. Such discontinuities can be also seen in a 1D radial plot (Fig. 2. Lazzati et al. (2009)), but the structure is different from our simulations. Such difference may be caused by the different code and resolution for the hydrodynamic calculations. 


\subsection{Thermal Radiation from Photosphere}

The thermal radiation from the photosphere is derived in post processing, from data taken every $1.0 \mathrm{~s}$ of the laboratory frame. In each snapshot of the hydrodynamic simulations, we find photosphere at unity optical depth for the Thomson scattering. The optical depth $(\tau)$ is defined as follows;

$$
\tau=\int_{x_{\mathrm{ph}}}^{\infty} \sigma_{\mathrm{T}} \Gamma(1-\beta \cos \theta) n d l
$$

where $\sigma_{\mathrm{T}}$ is the Thomson scattering cross section, $\Gamma\left(\equiv\left(1-\beta^{2}\right)^{-1 / 2}\right)$ is the Lorentz factor, $\beta$ is absolute value of velocity normalized by the speed of light, $\theta$ is the angle between the velocity vector $(\vec{\beta})$ and the line-of-sight (LOS), and $n$ is proper number density of the electron, $n \equiv 2 \rho / m_{\mathrm{He}}$, where $m_{\mathrm{He}}$ is the mass of helium atom. Though the progenitor includes many elements except hydrogen, we assume that all materials consist of fully ionized helium for simplicity. The expression in the integral includes the inverse of beaming factor $(\delta)$, i.e., $\Gamma(1-\beta \cos \theta)\left(\equiv \delta^{-1}(\theta)\right)$, to include the relativistic effect (Abramowicz et al. 1991). Here, we study the effect of the viewing angles, for an on-axis observer $\theta_{\mathrm{v}}=0$ and for off-axis observers, i.e., the angle between the jet axis and LOS, $\theta_{\mathrm{v}}=1^{\circ}$, and $2^{\circ}$.

Assuming an observer located at infinity, the isotropic luminosity of thermal radiation from the photosphere is evaluated as

$$
L_{\text {iso }}\left(\theta_{\mathrm{v}}\right)=a c \int \delta(\theta)^{4} T^{4} \cos \theta_{\mathrm{ph}} d S
$$

where $a$ is the radiation constant, $\theta_{\mathrm{ph}}$ is the angle between the LOS and the normal vector of the emission surface (Pe'er et al. 2007). The isotropic luminosity in the left hand side is considered by the observer time $\left(t_{\text {obs }}\right)$ which is related with the laboratory time $\left(t_{\text {lab }}\right)$ as $t_{\mathrm{obs}}=t_{\mathrm{lab}}+d / c$, where $d$ is the distance between each photosphere and the observer. The local temperature at the photosphere is evaluated as $T=(3 p / a)^{1 / 4}$, where $p$ is the thermal pressure of the fluid. The photosphere for $\theta_{\mathrm{v}}=0^{\circ}$ on the plane of the jet axis and the observer is shown by the solid lines in Fig. 1. Though we also plot the cases for $\theta_{\mathrm{v}}=5^{\circ}$, and $10^{\circ}$, we do not show light curves and spectrum for these cases due to too short duration at the observer frame (see, sec 3.3). The photospheres for each observer almost overlap at $t_{\text {lab }}=30 \mathrm{~s}$. As the jet advances, its density decreases. As a result, the photosphere shifts further inside the jet (bottom 4 panels in Fig. 1 at $t_{\text {lab }}=65$ and $90 \mathrm{~s}$ ). To an on-axis observer, the photosphere, in particular appears highly concave. 


\subsection{Light curves}

Hereafter we assume that the burst occurs at the redshift of $z=1$. Figure 2 shows the light curves for the observer at different viewing angles as a function of the observer time. The observer detects the radiation at different observer times, even if the radiation comes from the same laboratory time, due to the curved photosphere. We set $t_{\mathrm{obs}}=0$ as the burst trigger for each viewing angle. Though we integrate thermal radiation only of the whole computational laboratory time $\left(0<t_{\mathrm{lab}}<100 \mathrm{~s}\right)$ due to limited CPU time, the radiation will continue for some cases, especially on-axis case. Thus, we should stress that the light curves shown here are not completed yet, though the early phase of the light curve is valid. We need to follow longer timescales at laboratory time in the future.

The light curves exhibit time variability, i.e., for the observers $\theta=0^{\circ}$ and $1^{\circ}$ second $\left(10 \mathrm{~s}<t_{\mathrm{ob}}<22 \mathrm{~s}\right)$ and third $\left(t_{\mathrm{ob}}>22 \mathrm{~s}\right)$ phase after the first phases in the light curve which exhibits constant luminosity and continues for more than $32 \mathrm{~s}$. The feature of light curves is consistent with Lazzati et al. (2009) in which the case of $\theta=0^{\circ}$ is shown. Since the observer sees the skin of the jet at the beginning, the photosphere moves almost at the speed of light. The duration for the observer is about $1 / \gamma^{2}$ times as long as that for the laboratory frame, where $\gamma$ is the Lorentz factor of the motion for the photosphere $(\gamma \sim 10)$. The arrival time of the radiation concentrates within a few seconds for the observer which causes quick rise. As time passes, the density near head of the jet decreases due to the expansion of the jet. The beaming factor for the on-axis observer is very large, since the velocity vector is almost parallel to the jet axis. So, the distance between the forward shock and photosphere increases, i.e., Eq.1. The observer can see the region very deep inside the jet. The photosphere for the on-axis observer is concave as shown in bottom four panels of Fig. 1. Because of discontinuities in the Lorentz factor and density in the jet, the photosphere suddenly moves deeper inside the jet. The second phase caused by the detection of the internal shock $(\Gamma \sim 200)$ and beaming factor at the photosphere is more than 400 . The third phase is caused by the radiation from much deeper side. The Lorentz factor at the photosphere is smaller than that in second phase.

Figure 3 shows the photon number flux for an observer in the four energy bands as usually used in swift analysis. Figure 4 is same as Fig. 3, but different energy bands as

usually used in fermi analysis. For $\theta_{\mathrm{v}}=0^{\circ}$, only soft photons $(E \leq 100 \mathrm{keV})$ come in the beginning ( $t_{\mathrm{ob}} \leq$ a couple of seconds) since the Lorentz factor of the photosphere is still small. Then, high-energy photons follow. A few seconds time lag for high energy photons can be clearly seen in Fig, \for both on-axis observer $\left(\theta_{\mathrm{v}}=0^{\circ}\right)$ and off-axis observer $\left(\theta_{\mathrm{v}}=1^{\circ}\right.$, and $2^{\circ}$ ). 


\subsection{Spectrum}

Figure 5 shows the $\nu F_{\nu}$ plot for an observer $\left(\theta_{\mathrm{v}}=0^{\circ}, 1^{\circ}\right.$, and $\left.2^{\circ}\right)$. The spectrum is superposition of Plankian at local rest frame and each of them is boosted by the beaming factor. The interval of the whole observer time is integrated (top panel). The distribution is power law both below and above the peak energy. For $\theta_{\mathrm{v}}=0^{\circ}$, the spectrum has the peak energy at about $270 \mathrm{keV}$. A comparison with Fig. 4 shows that the spectrum is soft in the beginning, but becomes hard later. Further, as can be expected from Fig. 3 and 4 , the spectrum becomes softer for larger viewing angle cases $\left(\theta_{\mathrm{v}}=1^{\circ}\right.$ and $2^{\circ}$ cases $)$.

The spectrum below the peak energy has power law index with $2.3 \sim 2.6$ for all viewing angles. The indices are softer than that of single temperature plank distribution, see two bottom panels in Fig. 5 in which single temperature plank distribution, fitting to the peak energy and absolute value, is shown by thin dashed lines. Since the spectrum is superposition of different temperature Plank distribution, the power law index is $\sim 2.0$ near the peak energy and it continues to one tenth of the peak energy. The index is still harder than that of typical value of observed one, i.e., Band function (Band et al. 1993). The index for below the peak energy band in the spectrum of GRB090902B is about unity (Ryde et al. 2010). If we apply higher angular resolution for hydrodynamic calculation, the spectrum will consist of much more different local temperature $\left(T^{\prime}\right)$ and beaming factor $(\delta)$ contributions, resulting different observed temperature $\left(\delta T^{\prime}\right)$. This would make the spectrum softer. On the other hand, the index above the peak energy is softer than that of Band function. This suggests the non-thermal process is necessary to reproduce the entire GRB spectrum. We will investigate this non-thermal component in the near future.

\section{DISCUSSION and SUMMARY}

We have calculated the light curves and spectrum of the photospheric thermal radiation from GRB jets using 2D relativistic hydrodynamic simulations. We found that the thermal radiation from the photosphere of GRB jets is "observable" even for cosmologically distant GRBs. This component can be observed as a precursor or a thermal component in the prompt phase. The simulation needs further study to ascertain whether this component can be observed in the afterglow phase.

As for GRB090902B, the flux level of the thermal-like component reaches as high as $10^{4} \mathrm{keV} \mathrm{cm}^{-2} \mathrm{~s}^{-1}$ at high peak energy $\left(E_{\mathrm{pk}}\right)(\sim 300 \pm 100 \mathrm{keV})$ even though it is a distant GRB with $z=1.822$ (Ryde et al. 2010). If we put our simulated GRB at $z=1.822$, the flux level would be also about $10^{4} \mathrm{keV} \mathrm{cm}^{-2} \mathrm{~s}^{-1}$ but with lower peak energy $E_{\mathrm{pk}} \sim 190 \mathrm{keV}$. To 
explain the discrepancy between the peak energy of GRB090902B and our model by $\delta(\theta) T$ (Eq.(2)), this factor should be greater by a factor of 1.57. On the other hand, since the flux level is comparable between GRB090902B and our model, it is suggested that the value for $\delta(\theta)^{4} T^{4} d S$ is comparable (Eq.(2)). Thus we can deduce that $\left(\Gamma^{2} T^{4} r^{2}\right)_{\mathrm{GRB}} /\left(\Gamma^{2} T^{\prime 4} r^{2}\right)_{\text {Model }}$ will be of the order of unity. Here $\Gamma$ is the bulk Lorentz factor of the photosphere and $r$ is the radius of the photosphere. This is because $\delta(\theta)^{4} T^{4} d S$ can be rewritten as $2 \pi \delta(\theta)^{4} T^{4} r^{2} \Gamma^{-2}$ (beaming factor), and $\delta(\theta) \sim \Gamma$ for a face-on observer. Since $r \sim 2 \times 10^{12} \mathrm{~cm}$ and $\Gamma \sim 2 \times 10^{2}$ in our model, we can constrain the value of $(r / \Gamma)$ for GRB090902B as $4.1 \times 10^{9}$. This can be written as $\Gamma \sim 2.4 \times 10^{2}\left(r / 10^{12} \mathrm{~cm}\right)$ in GRB090902B. It is noted that the radius of the photosphere depends on many factors in a complicated way such as the power, initial Lorentz factor, and mass loading of the jet. In this analysis, the radius of the photosphere of GRB090902B is assumed to be of the order of $10^{12} \mathrm{~cm}$ like the simulation in this study.

There are many explanations for many GRBs not showing a clear thermal spectrum. The power-law component probably dominates the thermal component, for example Pe'er \& Ryde (2010). For all viewing angles the derived spectrum below the peak energy is power law with the indices $2.3 \sim 2.6$. Maybe this thermal component changes to a non-thermal one due to the scatterings with non-thermal, high-energy electrons. GRB jets might be usually dominated by the magnetic field component, suppressing photospheric emission (Zhang \& Pe'er 2009; Z Zhang \& Yan 2011). The strict requirement for collimation may also be the reason.

The time delay of hard photons shown in Figure 3 and 4 is of interest, because it may be related to the delayed onset observed in some bursts (e.g. GRB080916C, Abdo et al. (2009)). It should be noted that very high-energy emission may not always correlate with lower energy emission, depending how it is created (Pe'er et al. 2006; Giannios 2008; Lazzati \& Begelman 2010).

We thank Hirotaka Itoh and Hiroki Nagakura for useful discussions. We appreciate Katsuaki Asano for useful comments. We are grateful to an anonymous for constructive and insightful suggestions. This work is partly supported by the Grants-in-Aid of the Ministry of Education, Culture, Sports, and Science and Technology (MEXT) (19540236, 21018002 A.M.) and (19047004, 21105509 S.N.), Japan Society for the Promotion of Science (JSPS) (19104006, 19740139, 21540304 S.N.) and Grant-in-Aid for the Global COE Program 'The Next Generation of Physics, Spun from Universality and Emergence' from MEXT of Japan. J.A. is supported by Grant-in-Aid for JSPS Fellows. This work was carried out on NEC SX8, at YITP, Kyoto University, on the Space Science Simulator (NEC SX9) at JAXA, and on XT4 at CFCA at NAOJ. 


\section{REFERENCES}

Abdo, A. A. et al. Fermi collaborations 2009, Sci., 323, 1688

Abramowicz, M. A., Novikov, I. D., \& Paczynski, B. 1991, ApJ, 369, 175

Aloy, M. A., Müller, E., Ibáñez, J. M., Martí, J. M., \& MacFadyen, A. 2000, ApJ, 531, L119

Band, D., et al. 1993, ApJ, 413, 281

Blinnikov, S. I., Kozyreva, A. V., \& Panchenko, I. E. 1999, Astronomy Reports, 43, 739

Campana, S. 2006, Nature, 442, 1008

Giannios, D. 2006, A\&A, 457, 763

Ghirlanda, G., Bosnjak, Z., Ghisellini, G., Tavecchio, F., Firmani, C. 2007, MNRAS, 379, 73

Giannios, D. 2008, A\&A, 480, 305

Goodman, J. 1986, ApJ, 308, L47

Ioka, K., Murase, K., Toma, K., Nagataki, S., \& Nakamura, T. 2007, ApJ, 670, L77

Komissarov, S. S., \& Falle, S. A. E. G. 1997, MNRAS, 288, 833

Komissarov, S. S., \& Falle, S. A. E. G. 1998, MNRAS, 297, 1087

Lazzati, D., Morsony, B. J., \& Begelman, M. C. 2009, ApJ, 700, L47

Lazzati, D., Morsony, B. J., \& Begelman, M. C. 2010, ApJ, 717, 239

Lazzati, D., Begelman, M. C. 2010, arXiv:1005.4704

Li, Li-Xin. 2007, MNRAS, 380, 621

Liang, E., Zhang, B., Zhang, B.B., Dai, Z.G., 2006, arXiv:astro-ph/060565

MacFadyen, A. I., \& Woosley, S. E. 1999, ApJ, 524, 262

Mészáros, P., \& Rees, M. 2000, ApJ, 530, 292

Mizuta, A., Yamada, S., \& Takabe, H., 2004, ApJ, 606, 804

Mizuta, A., Yamasaki, T., Nagataki, S., \& Mineshige, S. 2006, ApJ, 651, 960 
Mizuta, A., \& Aloy, M. A. 2009, ApJ, 699, 1261

Mizuta, A., Kino, M., \& Nagakura, H. 2010, ApJ, 709, L83

Morsony, B. J., Lazzati, D., \& Begelman, M. C. 2007, ApJ, 665, 569

Morsony, B. J., Lazzati, D., \& Begelman, M. C. 2010, ApJ, 723, 267

Nagataki, S., Takahashi, R., Mizuta, A., \& Takiwaki, T. 2007, ApJ, 659, 512

Nagataki, S. 2009, ApJ, 704, 937

Pe’er, A., Mészáros, P., Rees, M. J. 2006, ApJ, 642, 995

Pe'er, A. et al. 2007, ApJ, 664, L1

Pe'er, A. 2008, ApJ, 682, 463

Pe'er, A., \& Ryde, F. 2010, arXiv:1008.4590

Piran, T. 1999, Phys. Rep., 314, 575

Rees, M., \& Mészáros, P. 2005, ApJ, 628, 847

Ryde. F., 2005, ApJ, 625, L95

Ryde. F. \& Re'er, A. 2009, ApJ, 702, 1211

Ryde, F., et al. 2010, ApJ, 709, L172

Soderberg, A. M., et al. 2008, Nature, 453, 469

Toma, K., Wu, X.-F., \& Mészáros, P. 2009, ApJ, 707, 1404

Toma, K., Wu, X.-F., \& Mészáros, P. 2010, arXiv:1002.2634

Woosley, S. E. 1993, ApJ, 405, 273

Woosley, S.E., Bloom, J. 2006, ARA\&A, 44, 507

Woosley, S. E., \& Heger, A., 2006, ApJ, 637, 914

Yoon, S.-C., \& Langer, N. 2005, A\&A, 443, 643

Yoon, S.-C., Langer, N. \& Norman, C. 2006, A\&A, 460, 199

Zhang, B., \& Pe'er, A. 2009, ApJ, 700, L65 
Zhang, W., Woosley, S. E., \& MacFadyen, A. I. 2003, ApJ, 586, 356

Zhang, W., Woosley, S. E., \& Heger, A. 2004, ApJ, 608, 365

Zhang, B.-B., et al. 2010, arXiv:1009.3338

Zhang, B., \& Yan, H. 2011, ApJ, 726, 90 

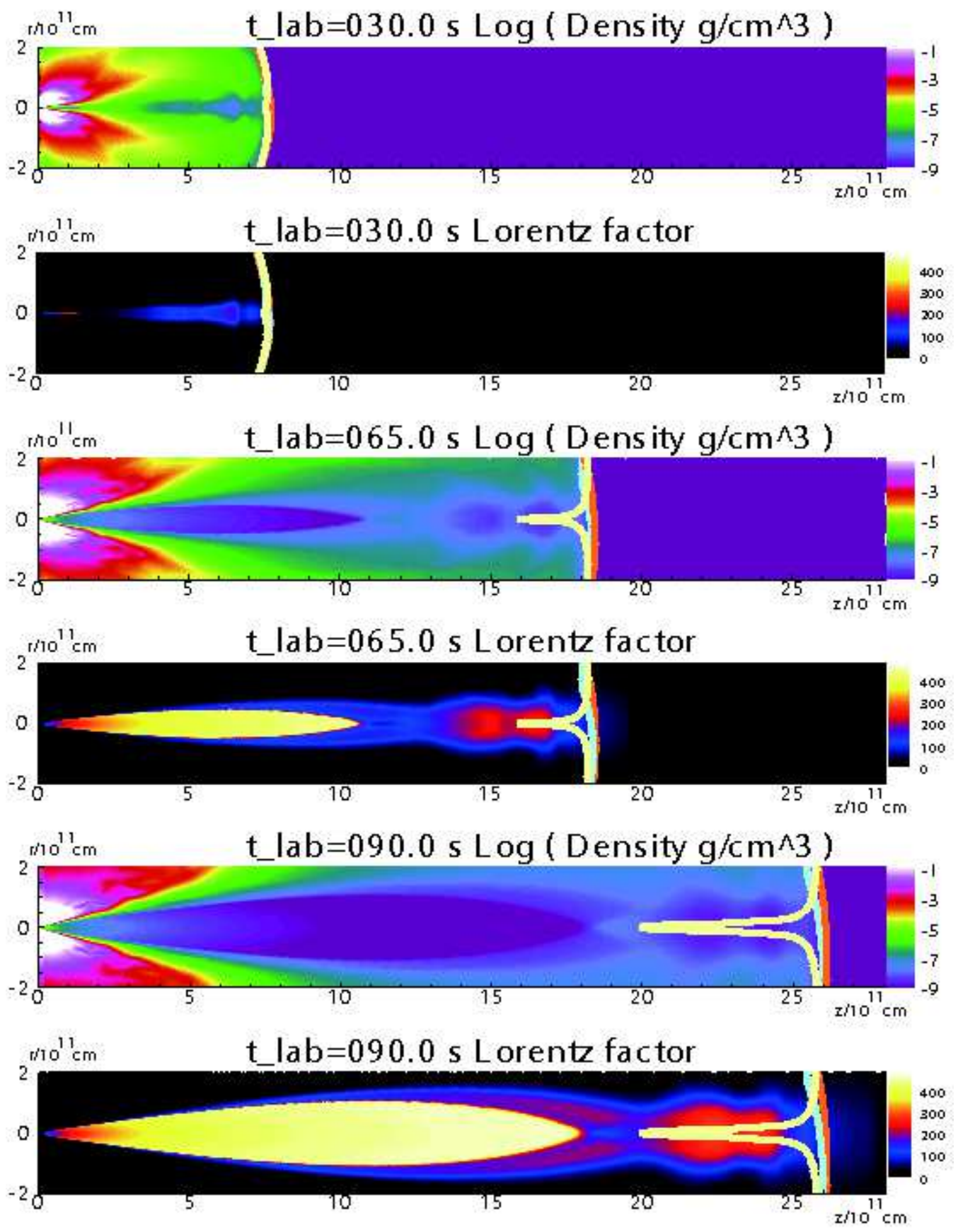

Fig. 1. - The density and Lorentz factor contours around the jet axis ( $z$-axis) at $t_{\text {lab }}=30$ (top two), 65 (middle two) and $90 \mathrm{~s}$ (bottom two). The photospheres for the observer at different viewing angles (yellow : $\theta=0$, aqua : $\theta=5^{\circ}$, and orange : $\theta=10^{\circ}$ ) are shown. The observer is at infinity and on $z$-axis $\left(\theta_{\mathrm{v}}=0\right)$ and at the right-top side $\left(\theta_{\mathrm{v}}>0\right)$. 




Fig. 2.- The light curves for the observer at different viewing angles, i.e., $\theta_{\mathrm{v}}=0^{\circ}($ solid $), 1^{\circ}$ (dashed), and $2^{\circ}$ (dotted). It is assumed that the burst occurs at the redshift of $z=1$. The duration of the radiation is longer for the on-axis observers. The light curve for an on-axis observer is bright and has time variability. We should stress the light curves still continues and the last part of the light curves for each viewing angle are not completed. 


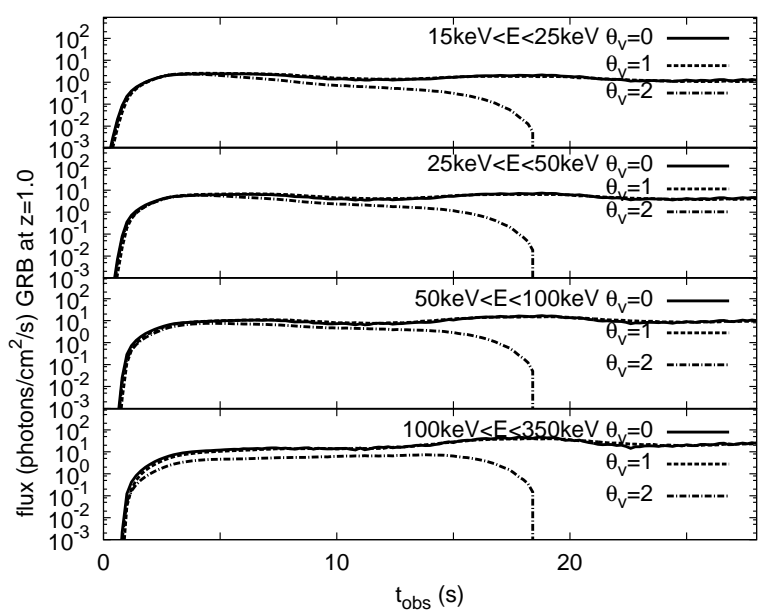

Fig. 3.- Photon number flux as a function of observer time for each energy bands (from top to bottom, $15 \mathrm{keV}<\mathrm{E}<25 \mathrm{keV}, 25 \mathrm{keV}<\mathrm{E}<50 \mathrm{keV}, 50 \mathrm{keV}<\mathrm{E}<100 \mathrm{keV}, 100 \mathrm{keV}<$ $\mathrm{E}<350 \mathrm{keV})$. It is assumed that the burst occurs at the redshift of $z=1$. The cases of three different viewing angles $\theta_{\mathrm{v}}=0^{\circ}, 1^{\circ}$, and $2^{\circ}$ are shown.

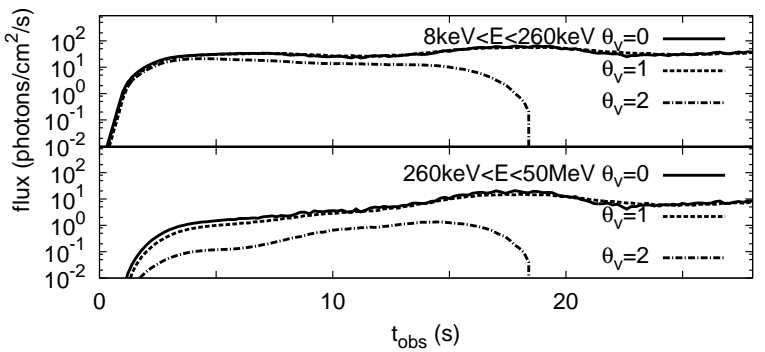

Fig. 4. - Same as Fig. 3, but different energy bands $8 \mathrm{keV}<\mathrm{E}<250 \mathrm{keV}$ (top) and $250 \mathrm{keV}<\mathrm{E}<50 \mathrm{MeV}$ (bottom). 




Fig. 5.- $\nu F_{\nu}$ plot for different viewing angles $\theta_{\mathrm{v}}=0^{\circ}, 1^{\circ}$, and $2^{\circ}$. Total time averaged spectrum are shown (top), and time averaged plots for two intervals for the on-axis observer are shown (middle and bottom). It is assumed that the GRB occurs at the redshift of $z=1$. Thin dashed lines shown in middle and bottom panels are single temperature Plankian distributions (peak energy and absolute value are fitted.) The power law indices below the peak energy are $2.3 \sim 2.6$. 